\title{
A Comprehensive Approach for Sub-Synchronous Resonance Screening Analysis Using Frequency scanning Technique
}

\author{
Mahmoud Elfayoumy ${ }^{1}$, Member, IEEE, and Carlos Grande Moran ${ }^{2}$, Senior Member, \\ IEEE
}

\begin{abstract}
The paper presents a comprehensive approach for sub-synchronous resonance (SSR) screening analysis using a developed frequency scanning tool capable of handling power networks with hundreds of buses. PTI's software packages like PSS/E and IPLAN programs were used for the development of the SSR tool. The frequency scanning technique scans for the sub-synchronous frequency range between $5 \mathrm{~Hz}$ to $59 \mathrm{~Hz}$ to determine the system driving point impedance (as a function of frequency) viewed from the neutral point of the generating unit under study. The proposed approach was applied to analyze the SSR phenomenon on several steam and gas driven turbine-generator plants in the northern part of the Western System Coordinating Council (WSCC) control area where several 500kV-transmission lines include series capacitor compensation. As a part of the study, credible contingencies that may lead to a network topology susceptible to SSR phenomenon are identified for proposed plants considered in the study.
\end{abstract}

Key Words: Capacitor Compensated Transmission Lines, Sub-synchronous resonance.

\section{Introduction}

Series capacitor compensation has been used widely in the AC transmission systems [1] as an economical alternative for different purposes such as increasing transfer capability through a particular interface, controlling load sharing among parallel lines, and enhancing transient instability [2]. However, their presence in the system may lead to the SSR phenomenon especially for the nearby generating plants that have a direct or a near radial connection to series capacitor compensated line(s).

Turbine-generator shaft failure and electrical instability at oscillation frequencies lower than the normal system frequency result from SSR. The two shaft failures at the Mohave Generating Station in Southern Nevada [3] led to the advancements in understanding the SSR phenomenon as well as explaining the interaction between series capacitor compensated lines and the torsion mode of steam turbinegenerators.

\footnotetext{
${ }^{1}$ M. Elfayoumy is a consultant with Power Technologies, Inc., NY 12301 USA (e-mail: Mahmoud.elfayoumy@shawgrp.com

${ }^{2}$ C. G. Moran is an executive consultant with Power Technologies, Inc., NY

12301 USA (e- mail: Carlos.Grande@shawgrp.com
}

IEEE Sub-Synchronous Resonance Working Group report [4] presented the basic theory, problem definition, analytical tools, testing, and countermeasures for mitigating the SSR effects. The report also discussed some related problems not caused by series capacitors such as device dependent synchronous oscillation (SSO) resulting from the interaction of a turbine-generator with fast acting controllers of power system components.

In an attempt to analyze the SSR phenomenon, several techniques were discussed in [5] to simulate and analyze the SSR phenomenon. The most common techniques are eigenvalue analysis, transient torque analysis, and frequency scanning. Eigenvalue analysis deals with self-excitation and accurately provides all the natural modes and damping or undamping of the coupled electrical and mechanical system. However, eigenvalue studies are relatively expensive and suited for a rather small size network. Transient torque analysis deals with transient shaft torque due to SSR. The frequency scanning technique involves the determination of the driving point impedance over the defined frequency range of interest as viewed from the neutral bus of the generator under study. Frequency scanning studies are relatively inexpensive for large networks involving hundred of buses. As it was indicated in [5], frequency-scanning approach is an effective way to screen out system conditions that are potentially hazardous from an SSR standpoint.

In this regard, this paper presents a comprehensive approach for SSR screening analysis using a developed frequency-scanning tool capable of handling power networks with hundreds of buses. The developed tool utilizes PTI's software packages such as PSS/E and IPLAN. The tools was used to analyze the SSR phenomenon on several steam and gas driven turbine-generator plants in the northern part of the WSCC control area where several 500kVtransmission lines include series capacitor compensation. The results of the analysis are presented and discussed

\section{Frequency Scanning Approach}

The frequency scanning-based approach for SSR analysis consists of two major modules as shown in Figure 1. 


\section{Module 1: Data manipulation and Network Reduction Module}

Since the effects of SSR are attenuated over distance, it is desirable to develop an equivalent of the network outside the area of which the study is to be performed. This will also decrease the computational time required to perform the frequency scanning in the second modules. Network reduction is only necessary for analysis of a large interconnected system. However, the tool is capable of analyzing the whole system if necessary. PSS/E package is used in this module.

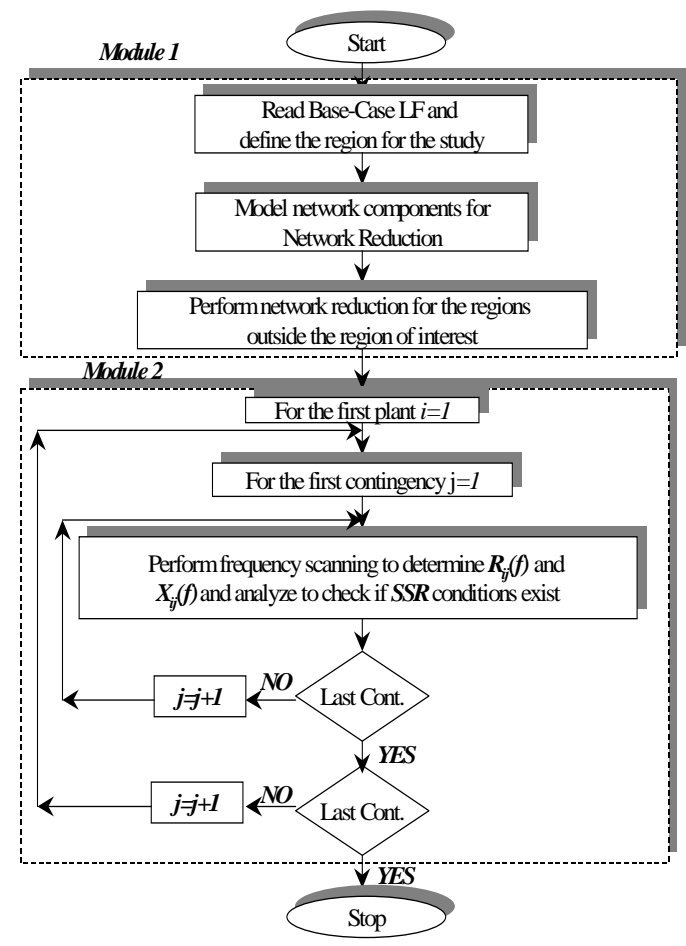

Figure 1. Frequency scanning-Based SSR Analysis Scheme

Several assumptions are made when modeling different network components as follows:

1. Generators are modeled as constant voltage source behind generator armature resistance and sub-transient reactance. An $\mathrm{X} / \mathrm{R}$ ratio of 125 is used in estimating the armature resistance if this data is not available.

2. Loads and shunt compensating elements are modeled as frequency-dependent admittances.

3. Transformers are represented as short equivalent lines, i.e. by lumped resistance and reactance between two buses.

4. Transformers phase shift angles are set to zero; any transformer impedance, which is a function of the phase shift, is set to its nominal value.

5. Transmission lines are represented as long lines (piequivalents) that include the effect of line charging currents. The reactive component of the series impedance in the transmission line model includes both inductive and capacitive (series capacitors) elements.

6. The HVDC stations and FACTS devices are not generally considered because they provide a limited amount of damping and do not modify the electrical network resonant frequencies. The exclusion of the HVDC terminals, then, provides conservative SSR results in that SSR instability would be reduced slightly by its inclusion. 7. Bus voltages are set to 1 p.u and zero phase angles.

With these assumptions, the reduced network outside of the region under study is built and ready for the frequency scanning in the second module.

\section{Module 2: Frequency scanning Module}

In this module, for each plant $i$ and for every contingency $j$, frequency scanning of the sub-synchronous frequency range between $5 \mathrm{~Hz}$ to $59 \mathrm{~Hz}$ determines the system driving point impedance $\left(Z_{i j}(f)\right)$, as a function of frequency, viewed from the neutral point of the generator unit under study. The next step is to check for the Induction Generator Effect and Torsional Interaction [5].

The condition of induction generator effect may occur when the electrical network, as viewed from the neutral of the unit under study, exhibits a series resonant condition at an electric frequency $\mathrm{f}_{\mathrm{e}}$, and the system resistance as seen from the neutral of the unit under study $\left(R_{i j}(f)\right)$ is smaller than the rotor resistance at the per unit slip frequency s given by

$$
\mathrm{s}=\frac{\mathrm{f}_{\mathrm{e}}-60}{\mathrm{f}_{\mathrm{e}}}(\mathrm{pu})
$$

Note that this per unit frequency will always be a negative number because $f_{e}$ is a sub-synchronous electric frequency. Thus, the rotor resistance (calculated based on unit I base MVA and voltage) the as seen from the neutral of the $\boldsymbol{i}^{\text {th }}$ generator will be negative and equal to

$$
\frac{r_{r i}}{s} \approx \frac{2}{s}\left(r_{2 i}-r_{1 i}\right)
$$

Where,

$r_{2 i}$ is the per unit negative sequence generator resistance $r_{1 i}$ is the per unit armature generator resistance

Torsional interaction may occur when a natural torsional frequency of the spring-mass torsional model of the turbinegenerator unit under study is very near $( \pm 1 \mathrm{~Hz})$ to a network electric resonant frequency. This is a sufficient but not necessary condition for torsional instability to occur. Torsional instability would occur if there is not sufficient electrical damping in the machine to damp out the SSR oscillations. Thus, the points in the frequency scanning plot of the system reactance that identify network configurations with SSR problem are those where the reactance is zero (series resonant point).

\section{Case Study}

The developed tool was applied to analyze the SSR phenomenon on several steam driven turbine-generator plants in the northern part of the Western System Coordinating Council (WSCC) area where several 500kV-transmission lines have series capacitor compensation. The study was performed to evaluate the SSR for credible contingencies that may lead to a network topology susceptible to SSR phenomenon.

For the purpose of including only those components that are of importance to the SSR screening study, a study system was created to include the $\mathrm{HV}$ and EHV transmission network in region under study and control areas with direct ties to that 
region. After Network reduction, the study system includes 712 buses, 147 loads, 1269 branches, 246 generators, 6 control areas and no HVDC terminals.

The generation dispatch specified in the original LF base case was kept in the SSR screening study. However, as part of the methodology used in the frequency scanning method a few number of units were set off-line to study the sensitivity of the electrical resonant frequencies to the number of units in the study system being out of service. Three proposed power plants were screened for SSR: two are combined cycle plants: Plant 1 and Plant 2; the third plant (Plant 3) is a steam plant. The number of generating units in Plant 1and Plant 3 is two while the number of units at Plant 2 is four.

The operating transmission voltages in the study system are $500 \mathrm{kV}, 345 \mathrm{kV}, 230 \mathrm{kV}$ and $115 \mathrm{kV}$. Branches at lower voltage levels were not included in the study system because they present a high impedance path to flow of SSR currents and thus they do not significantly affect the study results. Network, generation and loads outside the study area were reduced to short circuit static equivalents connected at the boundary buses.

Two contingency scenarios were considered when studying the SSR phenomenon for the different units in the three plants under study as follows:

Scenario 1: considers credible double contingency outages from a set of a double contingency list. The selected credible double contingency outages are chosen based on the system configurations that they create that make the plant under study to some how, either directly or near radially connected to series capacitor compensated line(s).

Scenario 2: determines the existence of a system configuration that may result in the onset of SSR for the each of the power plant under study. This critical configuration occurs when the plant is radially connected to a series capacitor compensated line and the electrical resonant frequency is the highest. This system configuration yields the lowest driving point impedance or highest system conductance. Thus, the methodology of contingency selection would require an achievement of the highest possible conductance at the highest possible electrical resonant frequency. Outages of components that shunt SSR currents away from the generator unit under study will reduce system conductance and have only a small effect on resonant frequency. This system configuration is observed in plants with multiples units. The more units are operated in parallel the smaller is the impact of the SSR currents flowing to the generator under study. Therefore, the worst operating condition for SSR in plants with multiple units is one when there is only a single unit in service. The results for the SSR study using the two contingency scenarios mentioned above for the three plants are presented in the next sections and discussed.

\section{A. SSR Analysis for Plant 1}

For the system configuration with contingency scenario1 and unit \#1 in service, there is no indication for the potential of induction generator effect. The same conclusion is drawn when unit \#2 is the only unit in service at Plant 1 . This system configuration does not show a potential for torsional interaction when one unit is in service and when both units are in service.

For the contingency scenario 2, the following results were obtained:

1. When either unit \#1 or unit \#2 is on line, there is no indication for induction generator effect SSR problems.

2. When unit \#1 is on line, there is a significant potential for torsional interaction if there is a torsional natural frequency close to $19.5 \mathrm{~Hz}$. The variation of the network conductance as seen from the neutral of unit \#1 is shown in Figure 2.

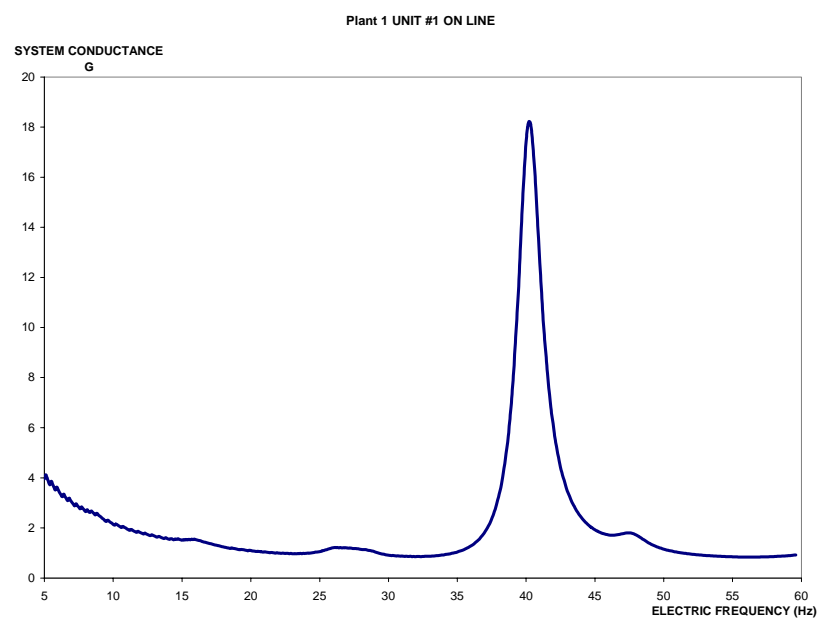

Figure 2. System Conductance by Unit \#1 For Near Radial Connection

3. When unit \#2 is on line, there is no indication for a potential torsional interaction. The system conductance observed at the electric frequency of $39.9 \mathrm{~Hz}$ is too small to indicate any strong torsional interaction between the electric network and the unit torsional system.

4. When both units are on line, there is a small shift to higher electric frequencies as seen by units \#1 and \#2. The new values for these frequencies are $40.7 \mathrm{~Hz}$ and 40 $\mathrm{Hz}$, respectively. The potential for torsional interaction observed for single operation of unit \#1 and unit \#2 is higher than for parallel operation since outages near and parallel to a generator will shunt SSR currents away from the generator by reducing system conductance.

\section{B. SSR Analysis for Plant 2}

For Plant 2, Units \#1 and \#3, and unit \#2 and unit \#4 operate in a combined cycle mode. Combustion turbines are furnished for units \#1 and \#2 while steam turbines are used in units \#3 and \#4.

For contingency scenario 1 with unit \#1 in service, there is no indication for the potential of induction generator effect. The same conclusion is drawn when unit \#3 is the only unit in service at Plant 2. As for torsional interaction in this scenario, there is a potential for torsional interaction at an electric 
frequency of $36 \mathrm{~Hz}$ when only unit \#1 is in service. When unit \#3 is the only unit in service the potential torsional interaction is negligible. The operating scenario when all units are in service shifts the potential for torsional interaction to a higher electric frequency, $36.7 \mathrm{~Hz}$, when viewed from unit \#1 and no problems with torsional interaction for unit \#3.

As for contingency scenario 2, the following results were obtained:

1. When only unit \#1 is in service, there is a potential for induction generator effect at an electric frequency of 33.7 $\mathrm{Hz}$ and $55.2 \mathrm{~Hz}$. The system resistance values at these two electrical resonant frequencies are 0.0024 p.u and 0.0075 p.u, respectively. Frequency scanning results are shown in Figure 3.

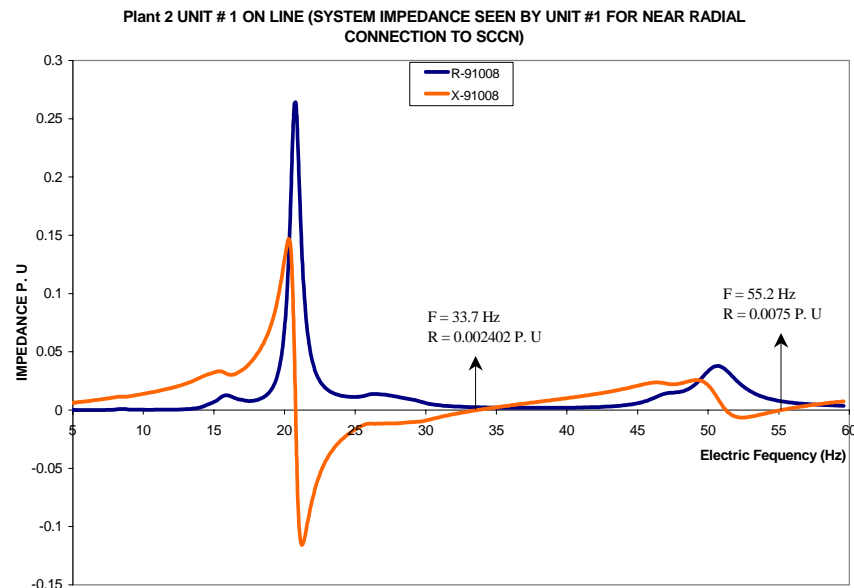

Figure 3. System Driving Point Impedance seen by Plant 2 unit \#1 for a Near Radial Connection (Units \#2, 3 and 4 are not in service).

2. When only unit \#3 is in service, there is no indication for a potential induction generator effect because unit \#3 does not see any network resonant condition in the subsynchronous range.

3. When only unit \#1 is in service, there is a potential for torsional interaction if unit \#1 has natural torsional frequencies close to $26.3 \mathrm{~Hz}$ and $4.8 \mathrm{~Hz}$. Potential torsional interaction at the electric frequency of $55.2 \mathrm{~Hz}$ (4.8 Hz torsional) is not an issue because the majority of combustion turbines exhibit sub-synchronous torsional frequencies in the range between $14 \mathrm{~Hz}$ to $35 \mathrm{~Hz}$. Figure 4 shows system conductance as a function of electrical frequency.

4. When only unit \#3 is in service, there is a potential for torsional interaction if unit \#3 has natural torsional frequencies close to $50.9 \mathrm{~Hz}$ and $9.8 \mathrm{~Hz}$ as shown in Figure 5.

5. The operating scenario of having all four units in service causes a small shift to higher frequencies. Also, outages near and parallel to a generator will shunt SSR currents away from the generator by reducing system conductance.

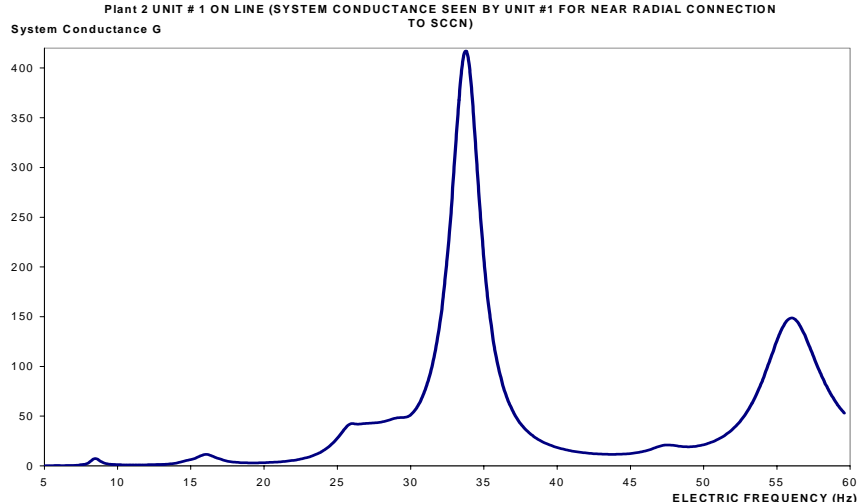

Figure 4. System Driving Point Conductance as seen from Plant 2 Unit \#1, Units 2,3, and 4 are not in service.

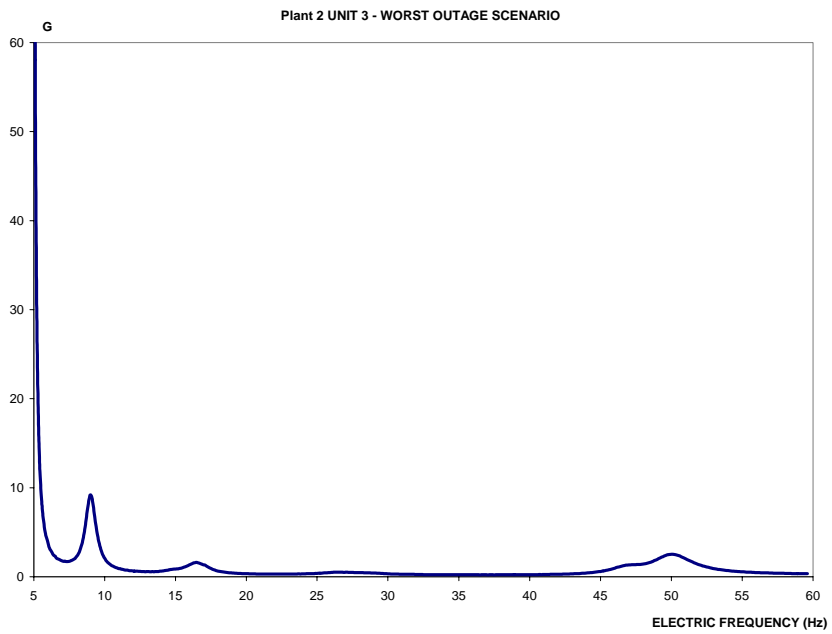

Figure 5. System Driving Point Conductance for Plant 2 Unit \#3.

6. The loss of several generators in the vicinity of Plant 2 does not change appreciably the electric resonant frequencies for any of the two system configurations and operating scenarios studied for this plant.

\section{SSR Analysis for Plant 3}

For contingency scenario1 with unit \#1 in service, there is no indication for the potential of induction generator effect. The same conclusion is drawn when unit \#2 is the only unit in service at Plant 3. This system configuration does not show a potential for torsional interaction when one unit is in service and when both units are in service.

For contingency scenario 2, there is no indication for the potential of induction generator effect. This system configuration, however, show a significant potential for torsional interaction if either unit \#1 or unit \#2 has a torsional frequency close to the electric frequency of $48.4 \mathrm{~Hz}$ as shown in Figure 6. 


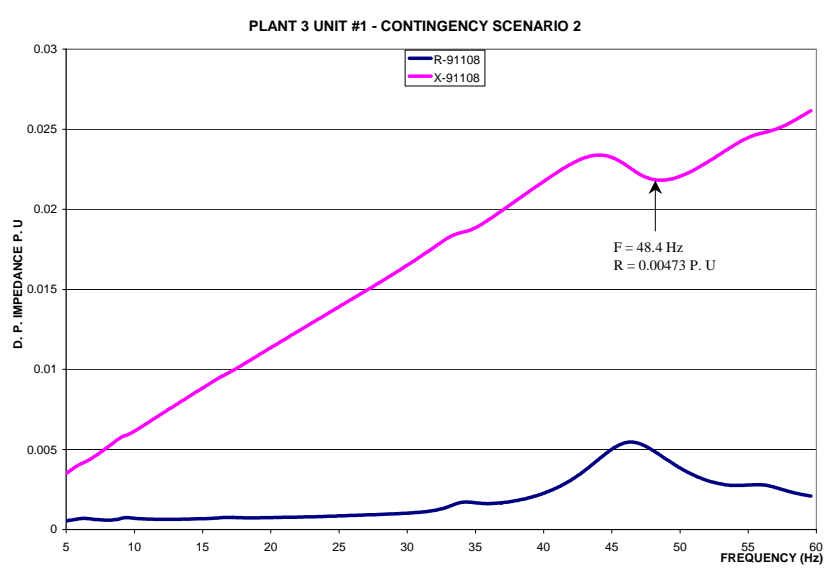

Figure 6. System Driving Point Impedance Seen by Unit \#1 for Plant 3

When both units are in service there is a small shift in electric frequency at which $(48.5 \mathrm{~Hz})$ there is potential for torsional interaction as shown in Figure 7.

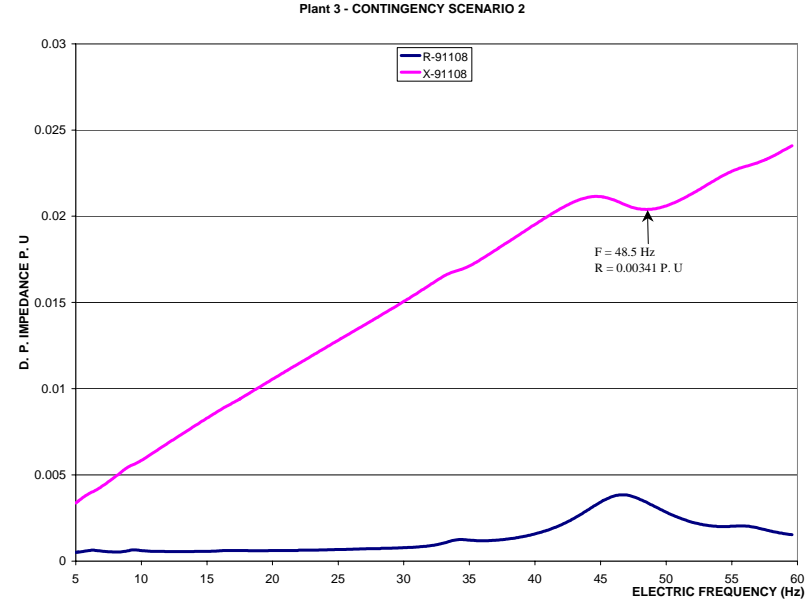

Figure 7. System Driving Point Impedance Seen by Unit \#1 for Plant 3 (Both units are on-line)

Outages near and parallel to a generator will shunt SSR currents away from the generator by reducing system conductance.

\section{Conclusion}

The paper presented a comprehensive approach for subsynchronous resonance (SSR) screening analysis using a developed frequency-scanning tool capable of handling power networks with hundreds of buses. PTI's software packages like PSS/E and IPLAN programs were used for the development of the SSR tool. The frequency scanning technique scans for the sub-synchronous frequency range between $5 \mathrm{~Hz}$ to $59 \mathrm{~Hz}$ to determine the system driving point impedance (as a function of frequency) viewed from the neutral point of the generating unit under study. The proposed approach was applied to analyze the SSR phenomenon on several steam and gas driven turbine-generator plants in the northern part of the Western System Coordinating Council (WSCC) control area where several $500 \mathrm{kV}$-transmission lines include series capacitor compensation. As a part of the study, credible contingencies that may lead to a network topology susceptible to SSR phenomenon are identified for the different plants considered for the study.

\section{References}

[1] "Series Capacitor Controls and Settings as countermeasures to Sub-Synchronous Resonance,” IEEE Sub-Synchronous Resonance Working Group of the System Dynamic Performance Subcommittee, IEEE Transactions on Power Apparatus and Systems, Vol. PAS-101, No. 6 June 1982.

[2] “Power System Stability and Control,” Prabha Kundur, EPRI Power System Engineering Series, 1993.

[3] J.W. Butler \& C. Concordia, "Analysis of Series Capacitors Application Problem,” AIEE Transactions, Vol. 56, pp. 975-988, 1937.

[4] “Reader's Guide to Sub-Synchronous Resonance,” IEEE Committee Report, IEEE Transactions on Power Systems, VOL. 7, No. 1, Feb. 1992.

[5] B. L. Agrawal, R. G. Farmer "Use of Frequency Scanning Techniques for Subs-Synchronous Resonance Analysis,” IEEE Transactions on Power Apparatus and Systems, VOL. PAS-98, No. 2 March/April 1979.

\section{Biographies}

Dr. Elfayoumy (M’02) received his B. Sc. and M. Sc. degrees in Electrical Power Engineering from Alexandria University, Alexandria Egypt, in 1991, and 1994 respectively with a final grade of Distinction with degree of Honor. He received his Ph.D. in Electrical Power Systems from Howard University, Washington, D.C. in May 2000. Currently he is a consultant at Power Technologies, Inc. working in the fields of transmission analysis and modeling. His current area of focus is in interconnection planning, reliability assessment, transfer limit calculations, fatal Flaw Analysis, Substation Screening Analysis, security constrained unit commitment, and securityconstrained dispatch. Dr. Elfayoumy is a member of IEEE and PES society. He is also a member of TAU BETA PI professional engineers honor society chapter of Washington DC and a member of Sigma Xi professional Scientific Research society. Dr. Elfayoumy published over 21 IEEE (IEE) journal and conference papers.

Dr. Grande-Moran (SM’1982) received a Diploma Engineer in Electrical and Mechanical Engineering from Universidad de El Salvador (UES) in 1974, a ME in Electrical Engineering (Power Systems) from Iowa State University in 1976, a ME in Systems Engineering from The University of Virginia in 1977, and a Ph.D. in Electrical Engineering (Power Systems) from Iowa State University in 1982. Currently, Dr. Grande-Moran is an Executive Consultant at Power Technologies, Inc. responsible for power system dynamics and controls, testing and monitoring of electric machinery, and power system planning. . Dr. Grande-Moran has over 20 years of experience in electric's generator design, power system dynamics, EMS systems, and stability. He is a Senior Member of the IEEE Power Engineering Society (PES). 